\title{
Event-Related Nociceptive Arousal Enhances Memory Consolidation for Neutral Scenes
}

\author{
Ulrike Schwarze, ${ }^{1}$ Ulrike Bingel, ${ }^{1,2}$ and Tobias Sommer ${ }^{1}$ \\ Departments of ${ }^{1}$ Systems Neuroscience and ${ }^{2}$ Neurology, University Medical Center Hamburg-Eppendorf, 20246 Hamburg, Germany
}

The superior memory for emotional events has been attributed to the beneficial effects of noradrenaline released into the amygdala attributable to arousal. Noradrenaline mediates the effects of different hormones and neurotransmitters, including adrenal stress hormones on consolidation (McGaugh, 2004; Roozendaal et al., 2009). The majority of human fMRI studies of the enhancement of emotional memories contrasted successful encoding of emotionally arousing and neutral stimuli (LaBar and Cabeza, 2006; Murty et al., 2010). Recently, it was highlighted that emotional stimuli elicit not only arousal but also intensify cognitive processes that contribute to the enhanced memory. In particular, the enhanced use of selective attention as well as the greater distinctiveness and semantic relatedness of emotional stimuli influence memory formation (Talmi et al., 2007a). The present study aimed to explore the effects of arousal on memory formation independent of these cognitive factors in an event-related manner. Arousal was induced by the application of a nociceptive stimulus briefly after the presentation of neutral scenes. The results show a purely arousal-driven memory enhancement for the neutral scenes that differs in critical aspects from the superior memory for emotional stimuli. In particular, the enhancement was only evident after consolidation and exclusively based on an increase in item familiarity but not recollection. Moreover, successful memory formation for stimuli followed by arousal was correlated with activity in the parahippocampal cortex but not the amygdala, as is the case for emotional stimuli.

\section{Introduction}

Emotionally arousing events are usually better remembered than neutral ones, an effect called the emotional enhancement of memory (EEM) (LaBar and Cabeza, 2006). According to the modulation hypothesis, the superiority of emotional memories is based on the arousal-induced release of stress hormones. Via noradrenaline (NA) released in the amygdala, these hormones mediate a more efficient consolidation (McGaugh, 2004). In addition, NA mediates the effects of other hormones and neurotransmitters on consolidation (Roozendaal et al., 2009). The majority of human studies of the EEM explore differences during encoding of emotionally arousing and neutral stimuli. Enhanced amygdala activity is consistently observed for successful encoding of emotional stimuli, supporting the crucial role of this structure in the EEM (Murty et al., 2010), although single-cell recordings indicate that the amygdala also responds to neutral stimuli (Kreiman et al., 2000). Moreover, consistent with the proposed effect of arousal on consolidation, the memory benefit for emotional stimuli is more pronounced after longer retention intervals (Sharot et al., 2007).

\footnotetext{
Received Sept. 2, 2011; revised Dec. 2, 2011; accepted Dec. 6, 2011.

Author contributions:U.S., U.B., and T.S. designed research;U.S. performed research;U.S. and T.S. analyzed data; U.S., U.B., and T.S. wrote the paper.

U.B. is supported by Federal Ministry of Education and Research Grant 01GQ0808. We thank Sabine Kastner for kindly providing the pictures of outdoor scenes, Matthias Gamer for assistance with the skin conductance analyses, and Jan Peters and Eszter Schoell for helpful comments on this manuscript.

Correspondence should be addressed to Ulrike Schwarze, Department of Systems Neuroscience, University Medical Center Hamburg-Eppendorf, Martinistrasse 52, 20246 Hamburg, Germany. E-mail: u.schwarze@ uke.uni-hamburg.de.

DOI:10.1523/JNEUROSCI.4497-11.2012

Copyright $\odot 2012$ the authors $\quad 0270-6474 / 12 / 321481-07 \$ 15.00 / 0$
}

However, superior memory for emotional stimuli is also observed immediately after encoding, which cannot be readily explained by more efficient consolidation. Therefore, the multifactor theory proposes that the EEM is not only driven by arousal but also by cognitive characteristics of emotional stimuli that are known to enhance memory performance (Maratos et al., 2000; Sharot and Phelps, 2004; Buchanan et al., 2006; Schmidt and Saari, 2007; Talmi et al., 2007a,b; Sommer et al., 2008). In addition to their semantic relatedness, the attraction of attention and enhanced initial processing of emotional stimuli contribute to the EEM (Schupp et al., 2006; Talmi et al., 2008).

The present study aimed to disentangle the effects of arousal from such cognitive characteristics of emotional stimuli on memory formation. Specifically, arousal was induced via the application of a nociceptive stimulus briefly after the presentation of neutral pictures. Similar to studies that explore the effect of posttraining interventions on memory consolidation (McGaugh, 2004; Andreano and Cahill, 2006; Yonelinas et al., 2011), the present approach thus aims to unravel the initial cognitive processing of neutral stimuli and the arousal elicited by the nociceptive stimulation in an event-related manner. By using this procedure, the cognitive-affective appraisal of the shock is expected to be primarily independent of the processing of the preceding neutral scene. Nociceptive arousal was induced by an electrical shock $50 \mathrm{~ms}$ after the presentation of neutral scenes in $50 \%$ of the events. In experiment 1 , the timescale of the effect of nociceptive arousal on memory formation was explored by contrasting two behavioral groups, tested immediately and $1 \mathrm{~d}$ after encoding. We expected a stronger impact of nociceptive arousal after a longer retention interval, reflecting enhanced consolida- 
tion. Based on the results of the first experiment, an fMRI study was conducted to compare the neural correlates of the effect with the activity patterns reported previously for emotional arousal.

\section{Materials and Methods}

Ethics approval for experiments 1 and 2 was obtained from the ethics committee of the medical association of Hamburg.

\section{Experiment 1}

Participants. Forty healthy volunteers participated in experiment 1 . They were randomly assigned to two behavioral groups (group 1, 6 males and 14 females; group 2, 13 males and 7 females), performing recognition immediately after encoding (day 1 group) or after a retention interval of $24 \mathrm{~h}$ (day 2 group).

Experimental task. During encoding, 80 unfamiliar neutral, nonarousing photos of outdoor scenes were shown for $800 \mathrm{~ms}$ each, containing either cars or people (Peelen et al., 2009). Participants were asked to classify each scene (i.e., cars vs people) as fast as possible by pressing the corresponding button. At $50 \mathrm{~ms}$ after stimulus offset, half of the scenes of each category were followed by an electrical shock consisting of a train of four $2 \mathrm{~ms}$ pulses that were delivered through an electrode on the left ventral forearm. In the following, the scenes followed by an electric shock will be termed scenes ${ }^{+ \text {shock }}$ and scenes not followed by a shock as scenes ${ }^{\text {no }}$ shock. The order of stimuli was pseudorandomized with the restriction that a maximum of five pictures of one condition (i.e., shock vs no shock) or one category (i.e., cars vs people) were presented in succession. The intensity of the electric stimulation was individually adjusted at the beginning of the experiment. During titration, subjects rated the intensity of each shock on a computerized visual analog scale (VAS), which ranged from 0 (stimulation not perceptible) to 100 (stimulation intolerable). The intensity representing a rating of 70 was delivered throughout the experiment. The VAS was also administered during encoding after the first, middle, and last shock of the actual experiment to test for habituation or sensitization. To suppress cognitive processing or elaborative encoding after stimulus offset, during the interstimulus interval (jittered $8-12 \mathrm{~s}$ ), participants had to determine via button press whether arrows presented for $800 \mathrm{~ms}$ each pointed to the left or the right (active baseline; Stark and Squire, 2001). Participants were informed about the upcoming memory test, but they were strongly encouraged to focus on the encoding and arrow-pointing tasks. The latter was motivated by specific timing requirements for the fMRI experiment.

In the day 1 group, recognition was tested briefly after encoding. Removing electrodes and giving instructions for the recognition test resulted in a temporal delay of $\sim 5 \mathrm{~min}$. In addition, the last five items shown were not presented within the first five old items in the recognition test to avoid recency effects. In the day 2 group, recognition was delayed by $\sim 24 \mathrm{~h}$. For the recognition task, old scenes were mixed with the same amount of new ones. Target and lures were randomly drawn from a larger set of pictures for each participant (Peelen et al., 2009). Responses were given self-paced on a 6-point confidence scale (from "high confidence old" to "high confidence new"), represented by six boxes displayed below each scene. In the case of an "old" response, participants were asked whether this scene was followed by shock during encoding using a yes/no forced-choice source memory task.

Skin conductance responses. Skin conductance was measured continuously throughout the experiment via $\mathrm{Ag} / \mathrm{AgCl}$ electrodes placed on the palm of the left hand. The signal was amplified using a CED 2502 amplifier and sampled at $10 \mathrm{~Hz}$ using a CED 1401 analog-to-digital converter (Cambridge Electronic Design). The first 0.5-6 s after each shock was checked individually to confirm that the shock led to a skin conductance response (SCR) that was unambiguously separable from the SCR elicited by the orienting response to the preceding picture. Then, the SCRs were quantified by subtracting the average skin conductance in the second before the stimulus onset from the maximum skin conductance within $4 \mathrm{~s}$ after stimulus onset. Data were $z$-transformed to account for interindividual differences in nociceptive reactivity. Because of data loss based on technical problems, only SCR data for 14 (day 1 group) and 13 (day 2 group) subjects are available.

\section{Experiment 2}

Participants. Twenty new participants ( 15 males) were scanned, using the paradigm established in experiment 1 (day 2-fMRI group in the following).

Experimental task. Based on the results of experiment 1, recognition was tested after a retention interval of $24 \mathrm{~h}$. Both encoding and recognition took place in the MR scanner, but only the encoding data are relevant here. Encoding was identical to experiment 1. During recognition, scenes were presented for $6 \mathrm{~s}$; the confidence scale was added after $2 \mathrm{~s}$ of scene presentation. The interstimulus interval was jittered between 3 and $6 \mathrm{~s}$. The source memory task was omitted.

Imaging. Functional MRI was performed on a $3 \mathrm{~T}$ system (Siemens Trio) with an echo planar imaging $\mathrm{T} 2^{\star}$-sensitive sequence in 40 contiguous axial slices ( $2 \mathrm{~mm}$ thickness with $1 \mathrm{~mm}$ gap; TR, $2.38 \mathrm{~s}$; TE, $25 \mathrm{~ms}$; flip angle, $70^{\circ}$; field of view, $192 \times 192 \mathrm{~mm}^{2}$; matrix, $64 \times 64$ ).

The image time series was slice-time corrected, realigned, and corrected for the interaction of motion and distortion using the "unwarp" toolbox of SPM8, normalized into standard anatomical space, and smoothed with a Gaussian kernel of $8 \mathrm{~mm}$ full-width at half-maximum using SPM8 (http://www.fil.ion.ucl.ac.uk/spm).

A first-level model was constructed for each subject on a voxel-byvoxel basis. In this model, the encoding-events were divided post hoc according to subsequent recognition performance (hits and misses for scenes $^{+ \text {shock }}$ and scenes $\left.{ }^{\text {no shock }}\right)$. The resulting four event categories were modeled as separate regressors by convolving a delta function at the time of shock onset with the canonical hemodynamic response function (hrf). It should be noted that creating the regressors based on the picture onsets did not change the results. In addition, the temporal and dispersion derivatives were included as separate regressors in the model. To show that the electrical shock was a potent arousing agent, scenes ${ }^{\text {tshock }}$ were contrasted with scenes ${ }^{\text {no shock }}$ regardless of memory performance (main effect of nociceptive arousal). Activity related to successful memory formation regardless of condition was identified by contrasting hits and misses (subsequent memory effect). Finally, the critical analysis was conducted by contrasting activity associated with successful encoding (i.e., the subsequent memory effects) in both conditions by applying the appropriate interaction contrast [the regressors corresponding to subsequent hits and subsequent misses for scenes ${ }^{\text {no shock }}$ and scenes ${ }^{+ \text {shock }}$ were weighted $(-1+1+1-1)$ ].

On the second level, the contrast images of the first-level analyses were tested with one-sample $t$ tests. To remove potential gender effects on neuronal activity (e.g., lateralization; Cahill, 2010), a covariate coding gender was included in the second-level models. Results of these analyses were considered significant at $p=0.05$ corrected for multiple comparisons at the entire scan volume and also within reduced search volumes of interests. The latter consisted of anatomical masks of different medial temporal lobe subregions, including the amygdala (Tzourio-Mazoyer et al., 2002; Amunts et al., 2005). The application of these masks was based on the pivotal role of these structures for memory and emotional processing (McGaugh, 2004; LaBar and Cabeza, 2006). For exploratory reasons, the threshold was relaxed to $p<0.001$ uncorrected and five contingent voxels at the entire scan volume. To test for differences in latency and width of the hrf between scenes ${ }^{+ \text {shock }}$ and scenes ${ }^{\text {no shock }}$, the parameter estimates for the temporal and dispersion derivatives were extracted at the peak voxel of the critical analysis and contrasted in a repeated-measures ANOVA outside of SPM.

\section{Results}

\section{Behavioral results}

Experiment 1

Performance in the encoding and the arrow-pointing tasks were highly accurate and did not differ between conditions in any group. Overall, the direction of $98 \%$ of the arrows was correctly indicated (day 1 group, $98.33 \pm 1.46 \%$; day 2 group, $98 \pm$ $1.34 \%)$. The scenes were correctly categorized in $95.93 \%$ of the trials (day 1 group, $96.37 \pm 2.74$ and $98 \pm 3.64 \%, t_{(19)}=1.71, p=$ 0.09 ; day 2 group, $95 \pm 4.44$ and $94.37 \pm 3.23 \%, t_{(19)}=0.89, p=$ 0.38 , for scenes $^{+ \text {shock }}$ and scenes ${ }^{\text {no shock }}$, respectively); $92 \%$ of all 
A

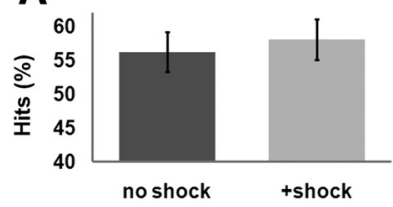

B

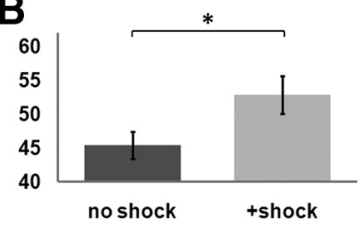

A
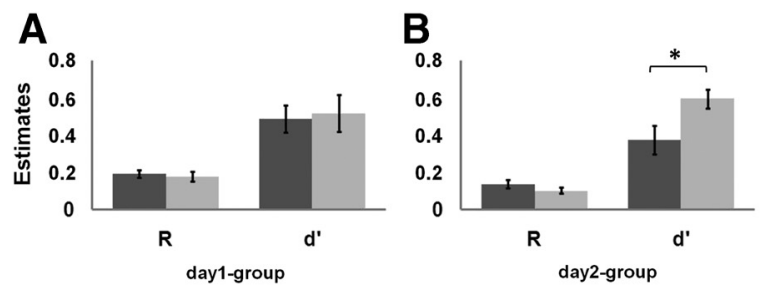
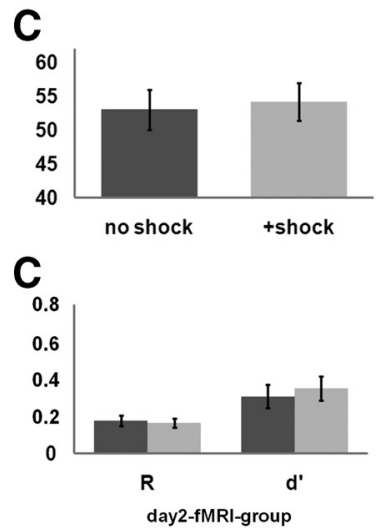

Figure 1. Top, Percentage of hits. Bottom, Estimates for recollection $(R)$ and familiarity $\left(d^{\prime}\right)$. A, Day 1 group. $\boldsymbol{B}$, Day 2 group. $\boldsymbol{C}$,

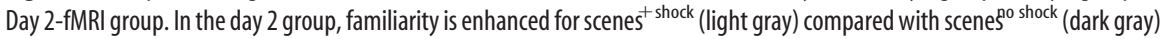
$\left({ }^{*} p<0.01\right)$. Whiskers reflect the SEM.

responses were executed during image presentation, i.e., before the electrical shock was potentially applied (median reaction time: day 1 group, $548 \pm 99.9 \mathrm{~ms}$; day 2 group, $542 \pm 60.1 \mathrm{~ms}$ ). These results imply that the initial cognitive processing was completed at the time of arousal induction.

The intensity rating of the electrical shock did not differ between groups (mean VAS day 1 group, $69 \pm 11.74$; mean VAS day 2 group, $\left.67.15 \pm 12.76 ; t_{(48)}=0.47, p=0.71\right)$. Moreover, it did not vary over time in any group (day 1 group, $65.6 / 69.3 / 72, F_{(2,38)}=2.2, p=0.12$; day 2 group, 65.3/67.1/69, $\left.F_{(2,38)}=1.14, p=0.32\right)$. In all subjects, the individual shocks elicited a highly reliable, time-locked SCR that was clearly separable from the orienting response to the preceding picture $(95.25 \pm 4.1 \%$ of all shocks). The condition $\times$ subsequent memory ANOVA revealed a main effect of condition in both groups (day 1 group, $F_{(1,14)}=7.35, p=0.02$; day 2 group, $F_{(1,12)}=6.45$, $p=0.02)$. Thus, arousal was enhanced after scenes ${ }^{+ \text {shock }}$ compared with scenes ${ }^{\text {no shock }}$. However, there was no significant interaction indicating similar SCR between subsequently remembered and forgotten scenes ${ }^{+ \text {shock }}$ (day 1 group, $F_{(1,13)}=0.007, p=0.97$; day 2 group, $\left.F_{(1,12)}=0.2, p=0.65\right)$. The SCR amplitude did not differ between the first and second half of the encoding session (day 1 group, $t_{(13)}=1.76, p=0.10$; day 2 group, $t_{(12)}=0.89, p=$ 0.39 ). Thus, neither habituation nor sensitization to the shock was detected.

Recognition performance was significantly above chance level in both conditions in both groups, indicated by corrected recognition scores, i.e., hits minus false alarms (day 1 group, $29.87 \pm 11.3 \%, t_{(19)}=11.78, p<0.001$; day 2 group, $23.5 \pm$ $\left.10.25 \%, t_{(19)}=10.42, p<0.001\right)$. The impact of condition on memory performance was analyzed in group $\times$ condition ANOVAs. For the corrected hit rate, a significant main effect of group (i.e., better memory performance in the day 1 group; $F_{(1,38)}=5.34, p=0.02$ ) and condition (i.e., better memory for scenes $\left.^{+ \text {shock}} ; F_{(1,38)}=8.43, p=0.006\right)$ was detected. Critically, the interaction resulted in a strong trend $\left(F_{(1,38)}=3.03, p=0.08\right)$ driven by the difference between scenes ${ }^{\text {no shock }}$ and scenes ${ }^{+ \text {shock }}$ in the day 2 group that was confirmed by post hoc Tukey's HSD tests $(p=0.01)$. This effect was further confirmed by separate group analyses on the hit rate. In the day 1 group, there was no evidence for a significant difference in recognition performance between conditions $\left(t_{(19)}=0.89, p=0.38\right.$; Fig. $1 A$, top $)$. In contrast, participants of the day 2 group recognized significantly more scenes followed by arousal $\left(t_{(19)}=3.06, p=0.006\right.$; Fig. $1 B$, top $)$.
A dual-process model of recognition memory was fitted to the confidence ratings to derive estimates of recollection $(R)$ and familiarity $\left(d^{\prime}\right)$ (Yonelinas et al., 1998). For $R$, a significant main effect of group, i.e., a stronger contribution of $R$ in the day 1 group $\left(F_{(1,38)}=5.93, p=0.01\right)$, a weak trend for the main effect of condition $\left(F_{(1,38)}\right.$ $=2.54, p=0.11)$, and no interaction $\left(F_{(1,38)}=0.51, p=0.47\right)$ was revealed. For $d^{\prime}$, a significant main effect of condition $\left(F_{(1,38)}=7.78, p=0.007\right)$ and an interaction was detected $\left(F_{(1,38)}=4.71, p=\right.$ $0.03)$. Post hoc tests revealed that $d^{\prime}$ differed in the day 2 group only, i.e., that $d^{\prime}$ was significantly higher for scenes followed by arousal $(p=0.006)$. Again, groups were also analyzed separately. In the day 1 group, $d^{\prime}$ did not differ between conditions $\left(t_{(19)}=0.40, p=0.69\right.$; Fig. $1 \mathrm{~A}$, bottom). In the day 2 group, $d^{\prime}$ was significantly higher for scenes followed by arousal $\left(t_{(19)}=4.04, p<0.001\right.$; Fig. $1 B$, bottom $)$.

Finally, corrected hit rates of the source memory task were at chance level for both groups, indicating that recognition of scenes followed by arousal was not supported by contextual cues (day 1 group, $-2.56 \pm 17.36, t_{(19)}=-0.68, p=0.5$; day 2 group, $\left.-0.9 \pm 13.82, t_{(19)}=-0.29, p=0.77\right)$.

\section{Experiment 2}

Performance in the encoding and arrow-pointing tasks of the day 2-fMRI group was correct and fast, similar to the behavioral groups (arrows, $98 \pm 1.44 \%$ correct; classification of images, $94.2 \pm 19.6 \%$ scenes $^{+ \text {shock }} ; 93.2 \pm 17.77 \%$ scenes $^{\text {no shock }}$ correct; median reaction time, $624.7 \pm 85.1 \mathrm{~ms})$. Again, performance was not influenced by the electrical shock $\left(t_{(19)}=0.25, p=\right.$ 0.8 ). Arousal did not differ from the behavioral groups (mean VAS, $\left.67.4 \pm 13.81, F_{(2,57)}=1.53, p=0.22\right)$ and was constant across the scanning session (VAS ratings, 66.1/67.9/68.3, $\left.F_{(2,38)}=1.67, p=0.2\right)$.

The corrected hit rate was significantly above chance level $\left(20.15 \pm 10.85 \%, t_{(19)}=10.24, p<0.001\right)$. However, there was no significant difference in the recognition performance for scenes $^{+ \text {shock }}$ and scenes ${ }^{\text {no shock }}\left(t_{(19)}=0.49, p=0.62\right.$; Fig. $1 C$, top). Also, $R$ and $d^{\prime}$ did not show a significant difference between conditions $\left(R, t_{(19)}=0.47, p=0.63 ; d^{\prime}, t_{(19)}=0.69, p=0.49\right.$; Fig. $1 C$, bottom). A condition $\times$ group ANOVA was computed to compare recognition performance between the day 2-fMRI group and the day 2 group. The analysis of the corrected hit rate showed a trend toward significance for the interaction effect $\left(F_{(1,38)}=3.55, p=0.06\right)$. In addition, the main effect of condition reached significance $\left(F_{(1,38)}=6.55, p=0.01\right)$, whereas the main effect of group was not significant $\left(F_{(1,38)}=1.79, p=0.18\right)$. The condition $\times$ group ANOVA with $R$ as dependent variable did not reveal significant effects (main effect condition, $F_{(1,38)}=2.39$, $p=0.12$; interaction, $\left.F_{(1,38)}=0.68, p=0.41\right)$ but only a trend toward significance for the main effect group $\left(F_{(1,38)}=2.91, p=\right.$ 0.09 ), indicating a greater rate of recollected responses of the day 2 -fMRI group. The condition $\times$ group ANOVA with $d$ ' as dependent variable showed a significant main effect of condition $\left(F_{(1,38)}=10.44, p=0.002\right)$, i.e., more familiarity-based recognition for scenes ${ }^{+ \text {shock }}$, a significant interaction $\left(F_{(1,38)}=4.84, p=\right.$ $0.03)$, and a trend toward significance for the main effect of group $\left(F_{(1,38)}=3.61, p=0.06\right)$, i.e., more familiarity-driven recogni- 
tion in the day 2 group. Tukey's HSD post hoc test revealed significant memory differences between scenes $^{+ \text {shock }}$ and scenes ${ }^{\text {no shock }}$ in the day 2 group.

\section{Imaging results}

During encoding, the main effect of arousal was associated with activity in the bilateral amygdala $[x y z=(28,2,-28)$, $Z=4.31 ; x y z=(-24,-4,-14), Z=$ 3.38; Fig. 2], the right secondary somatosensory $[x y z=(40,-16,18), Z=5.99]$, bilateral insular $[x y z=(-38,-2,-6)$, $Z=5.79 ; x y z=(40,2,-8), Z=5.47]$, and the left lateral parietal $[x y z=(-54$, $-54,18), Z=5.44$; Fig. 2] cortices. The lower exploratory threshold of $p<0.001$ uncorrected was survived also by peaks in the right temporal-parietal junction $[x y z=(60,-20,22), Z=5.65]$ and the left orbitofrontal cortex $[x y z=(-22,36$, $-14), Z=4.65]$.

Successful memory formation across encoding conditions was correlated with enhanced activity in the right hippocampus $[x y z=(24,-14,-10), Z=4.05, p=$ 0.011 ] and the bilateral fusiform gyrus $[x y z=(-24,-38,-14), Z=3.94 ; x y z=$ $(32,-40,-10), Z=3.77]$. At the lower threshold, the bilateral posterior hippocampi $[x y z=(32,-38,-10), Z=3.88 ; x y z=$ $(-14,-42,2), Z=3.56]$ and the left lingual gyrus $[x y z=(-14$, $-46,4), Z=3.92$ ] also showed significant activity.

Successful memory formation for scenes ${ }^{+ \text {shock }}$ compared with scenes ${ }^{\text {no shock }}$ was represented by enhanced activation of the right posterior parahippocampus $[x y z=(22,-34,-12), Z=3.72$, $p=0.023$; Fig. 3]. The lower threshold revealed activity in the right fusiform gyrus $[x y z=(36,-34,-14), Z=3.58]$, the left lingual gyrus $[x y z=(-20,-90,-6), Z=3.36]$, the right anterior cingulate $[x y z=(42,-10,40), Z=3.42]$, and the bilateral postcentral gyri $[x y z=(42,-10,40), Z=3.42 ; x y z=(-48,-12$, $38), Z=3.62]$. No such differential effect was observed in the amygdala even after lowering the statistical threshold (uncorrected $p<0.01)$. The temporal and dispersion derivative in the significant voxel did not differ significantly, indicating that neither the latency nor the width of the hrf was affected by the electric shock after stimulus offset.

\section{Discussion}

Experiment 1 revealed that memory was enhanced for scenes ${ }^{+ \text {shock }}$ not immediately but only after a $24 \mathrm{~h}$ retention interval, which is consistent with an effect of arousal on consolidation. Moreover, the superior memory was solely driven by familiarity, which is contrary to the typical increase in recollection for emotional stimuli. Experiment 2 showed that successful encoding for scenes ${ }^{+ \text {shock }}$ compared with scenes ${ }^{\text {no shock }}$ was mediated by activity in the right parahippocampal cortex. The SCR and amygdala activity correlated with the nociceptive stimulation but not with successful memory formation, which is in contrast to the findings of studies using emotional stimuli. In the following, we will discuss the present findings in relation to results from emotional arousal studies to elucidate the processes underlying the EEM.

\section{Behavioral performance}

Studies of the EEM that use emotional stimuli and sensitive memory tests observe an effect of arousal not only after longer retention intervals but also immediately after encoding. According to the multifactor theory, this immediate EEM is based on cognitive characteristics of emotional stimuli rather than on arousal (Talmi et al., 2007a). In the present study, scenes probed for memory did not differ with respect to such cognitive factors, e.g., distinctiveness or semantic relatedness. Moreover, nociceptive arousal was applied after the initial processing of individual scenes and could not result in the attraction of selective attention to specific scenes. Therefore, the current findings support the multifactor theory because only arousal and not the cognitive processing differed between conditions and only a delayed memory enhancement was observed.

In contrast to the memory enhancement for emotionally arousing stimuli, the memory enhancement by nociceptive arousal was exclusively driven by familiarity but not recollection (Kensinger and Corkin, 2003; Sharot et al., 2007; Sharot and Yonelinas, 2008). Because recollection specifically benefits from attention during encoding, this finding can be attributed to the similar initial processing in both conditions of the current study (Yonelinas, 2001; Kensinger et al., 2003). This interpretation is supported by the familiarity-driven memory enhancement for neutral stimuli in studies that induced arousal after a blocked encoding phase (McCullough and Yonelinas, 2011; Yonelinas et al., 2011). Together, the absence of an immediate memory effect and an increase in recollection in the present study suggest that the effects on these variables in studies using emotional stimuli rather result from differences in the initial processing than from pure arousal.

Unexpected at first, the day 2-fMRI group did not show an effect of arousal on memory performance. In particular, encoding and retrieving in the scanner had a beneficial effect on the hit rate for all scenes and not only on the memory for scenes ${ }^{+ \text {shock }}$. 
This observation could be explained by differences in the experimental settings. On the one hand, the restricted response time window inside the scanner might have led to the omission of slow, low confidence responses. On the other hand, participants are more motivated and focused on the task in an fMRI experiment because the extensive setup emphasizes the importance of their performance and there is less visual distraction. The scanner itself also constitutes a highly distinct and congruent context during encoding and retrieval that results in greater contextdependent memory (Smith and Vela, 2001; Törnqvist et al., 2006). The unique MRI-scanning experience might have led to additional incidental reactivation of both scenes ${ }^{+ \text {shock }}$ and scenes ${ }^{\text {no shock }}$ during the retention interval. The slight increase in recollection in the fMRI group would be consistent with these interpretations.

In addition, the MRI-scanning situation has been associated with the systemic secretion of adrenal stress hormones (Tessner et al., 2006; Eatough et al., 2009; Peters et al., 2011). Elevated cortisol levels in studies that used only neutral material resulted in improved memory performance (Andreano and Cahill, 2006; Beckner et al., 2006; Kukolja et al., 2008). The effect of arousal in the day 2 group of experiment 1 was presumably driven by centrally released NA and could not be attributable to adrenal hormones that are too slow to account for event-related effects (Strange et al., 2003; Roozendaal et al., 2009). Together, the beneficial effects of the fMRI setup on memory performance for all scenes might have masked the more subtle effects of poststimulus arousal. Moreover, when NA levels are elevated in addition to cortisol, there is no additive effect on memory performance (van Stegeren et al., 2010; Kukolja et al., 2011). Contrary to the neutral stimuli in the current study, the memory for emotional stimuli is enhanced in fMRI settings as well. Because the superior memory for emotional stimuli is also based on cognitive factors, it might not be as easily masked by other variables.

\section{Functional MRI data}

There was a stronger correlation for activity in the right parahippocampal cortex with successful memory formation for scenes $^{+ \text {shock }}$ than for scene $e^{\text {no shock }}$. Thus, nociceptive arousal enhances activity in an area that is engaged in the initial processing of scenes (Epstein et al., 2003). Recently, it has been suggested that the parahippocampal cortex is also involved in the encoding of contexts (Diana et al., 2007). However, because of the scenic stimulus material and the exclusive increase in familiarity-driven, acontextual recognition in the present setting, the parahippocampal activity presumably reflects scene processing (Doeller and Kaplan, 2011). An activity increase in this area has also been reported for successful encoding of emotionally arousing information (Murty et al., 2010). Therefore, this activity might reflect a common, arousal-induced process that does not depend on the initial processing based on the cognitive characteristics of emotional stimuli. The differential activity in the parahippocampal cortex was not accompanied by more efficient consolidation in the day 2-fMRI group. However, it reflects processing differences attributable to poststimulus arousal that are most likely related to the observed memory differences in the day 2 group outside of the scanner.

In the present experiment, bilateral amygdala activity and SCR were correlated with the electrical shock but did not show a systematic relationship with subsequent memory performance. In contrast, fMRI studies consistently report greater amygdala activity during successful encoding of emotionally arousing stimuli and contexts (Erk et al., 2005; Murty et al., 2010). Also, auto- nomic measures are typically correlated with successful memory formation for emotionally arousing stimuli (Anderson et al., 2006; Buchanan et al., 2006; Abercrombie et al., 2008). Emotional stimuli are characterized by a great variability in subjective arousal, and highly arousing stimuli lead to more pronounced autonomic responses and more amygdala activity and are also more likely to be remembered (Lang et al., 1993; Phan et al., 2004; van Stegeren et al., 2005). On the contrary, the nociceptive stimulation in the present experiment was physically and subjectively constant throughout the experiment. The small fluctuations in the sensory processing and affective appraisal of the shock did not lead to significant differences in SCR and amygdala activity, which are both directly related to memory formation.

However, parahippocampal activity correlated with memory for scenes ${ }^{+ \text {shock }}$ despite the constancy of arousal. This leads to the question of how constant arousal after the initial cognitive processing might tag specific events for a more efficient consolidation. Nociceptive stimuli activate the central noradrenergic system in the locus ceruleus (LC) directly and also indirectly via the centromedial amygdala that allocates attention to relevant stimuli (Van Bockstaele et al., 2001; Gao et al., 2004; Mosher et al., 2010). This results in NA release in the projection areas of the LC, for example, the basolateral amygdala, the hippocampus, and also the parahippocampus (Joyce et al., 1992; Berridge and Waterhouse, 2003). NA modulates activity in the basolateral amygdala and also mediates an increase in cortical neuronal responsiveness as well as in cortical synaptic plasticity (Mondaca et al., 2004; Flores et al., 2010; Tully and Bolshakov, 2010). Thus, the activation of the central noradrenergic system could affect processing in the parahippocampal cortex directly via afferents from the LC and/or indirectly via the basolateral amygdala (Suzuki, 1996).

The relatively constant (as reflected in the SCRs) modulatory input from the LC reaches the parahippocampal cortex during the initial neural processing of the stimuli because the shape of the BOLD response (i.e., its temporal and dispersion derivatives) does not indicate a later or prolonged neuronal processing. The increased parahippocampal responsiveness and plasticity might directly interact with stimulus-specific variability in the neural processing of the scenes. This increase in parahippocampal responsiveness increases the likelihood that activity or synaptic efficacy reaches a level that supports subsequent consolidation, for example, by the conversion from early into late LTPs, as formulated by the synaptic tagging hypothesis (Redondo and Morris, 2011). Whether the basolateral amygdala is involved in modulating parahippocampal responsiveness or whether its constant activity merely reflects the affective aspect of the nociceptive stimuli cannot be decided based on the data.

In conclusion, the present data show that event-related, arousal-induced enhancement of consolidation can occur independently of the initial processing of stimuli. This behavioral effect was demonstrated outside the MR scanner and was possibly masked when encoding took place inside the MR-scanner environment. Our data suggest that this behavioral effect may be based on centrally released NA-modulating responsiveness or synaptic efficacy in cortical areas involved in the processing of stimuli. However, these processes do not result in an immediately enhanced memory and an increase in recollection, as it is seen for emotional stimuli. Therefore, the data imply that the immediate EEM and the increase in recollection in studies using emotionally arousing stimuli might rely on enhanced initial processing, which is triggered by evaluative processes in the amygdala (Liddell et al., 2005; Vuilleumier, 2005). 


\section{References}

Abercrombie HC, Chambers AS, Greischar L, Monticelli RM (2008) Orienting, emotion, and memory: phasic and tonic variation in heart rate predicts memory for emotional pictures in men. Neurobiol Learn Mem 90:644-650.

Amunts K, Kedo O, Kindler M, Pieperhoff P, Mohlberg H, Shah NJ, Habel U, Schneider F, Zilles K (2005) Cytoarchitectonic mapping of the human amygdala, hippocampal region and entorhinal cortex: intersubject variability and probability maps. Anat Embryol (Berl) 210:343-352.

Anderson AK, Yamaguchi Y, Grabski W, Lacka D (2006) Emotional memories are not all created equal: evidence for selective memory enhancement. Learn Mem 13:711-718.

Andreano JM, Cahill L (2006) Glucocorticoid release and memory consolidation in men and women. Psychol Sci 17:466-470.

Beckner VE, Tucker DM, Delville Y, Mohr DC (2006) Stress facilitates consolidation of verbal memory for a film but does not affect retrieval. Behav Neurosci 120:518-527.

Berridge CW, Waterhouse BD (2003) The locus coeruleus-noradrenergic system: modulation of behavioral state and state-dependent cognitive processes. Brain Res Brain Res Rev 42:33-84.

Buchanan TW, Etzel JA, Adolphs R, Tranel D (2006) The influence of autonomic arousal and semantic relatedness on memory for emotional words. Int J Psychophysiol 61:26-33.

Cahill L (2010) Sex influences on brain and emotional memory: the burden of proof has shifted. Prog Brain Res 186:29-40.

Diana RA, Yonelinas AP, Ranganath C (2007) Imaging recollection and familiarity in the medial temporal lobe: a three-component model. Trends Cogn Sci 11:379-386.

Doeller CF, Kaplan R (2011) Parahippocampal cortex: translating vision into space. Curr Biol 21:R589-R591.

Eatough EM, Shirtcliff EA, Hanson JL, Pollak SD (2009) Hormonal reactivity to MRI scanning in adolescents. Psychoneuroendocrinology 34:1242-1246.

Epstein R, Graham KS, Downing PE (2003) Viewpoint-specific scene representations in human parahippocampal cortex. Neuron 37:865-876.

Erk S, Martin S, Walter H (2005) Emotional context during encoding of neutral items modulates brain activation not only during encoding but also during recognition. Neuroimage 26:829-838.

Flores O, Núñez H, Pérez H, Morgan C, Soto-Moyano R, Valladares L, Burgos H, Olivares R, Hernández A (2010) $\beta$-Adrenoceptor blockade depresses molecular and functional plasticities in the rat neocortex. Brain Res Bull 82:284-288.

Gao YJ, Ren WH, Zhang YQ, Zhao ZQ (2004) Contributions of the anterior cingulate cortex and amygdala to pain- and fear-conditioned place avoidance in rats. Pain 110:343-353.

Joyce JN, Lexow N, Kim SJ, Artymyshyn R, Senzon S, Lawrence D, Cassanova MF, Kleinman JE, Bird ED, Winokur A (1992) Distribution of betaadrenergic receptor subtypes in human post-mortem brain: alterations in limbic regions of schizophrenics. Synapse 10:228-246.

Kensinger EA, Corkin S (2003) Memory enhancement for emotional words: are emotional words more vividly remembered than neutral words? Mem Cognit 31:1169-1180.

Kensinger EA, Clarke RJ, Corkin S (2003) What neural correlates underlie successful encoding and retrieval? A functional magnetic resonance imaging study using a divided attention paradigm. J Neurosci 23:2407-2415.

Kreiman G, Koch C, Fried I (2000) Category-specific visual responses of single neurons in the human medial temporal lobe. Nat Neurosci 3:946-953.

Kukolja J, Thiel CM, Wolf OT, Fink GR (2008) Increased cortisol levels in cognitively challenging situations are beneficial in young but not older subjects. Psychopharmacology (Berl) 201:293-304.

Kukolja J, Klingmüller D, Maier W, Fink GR, Hurlemann R (2011) Noradrenergic-glucocorticoid modulation of emotional memory encoding in the human hippocampus. Psychol Med 41:2167-2176.

LaBar KS, Cabeza R (2006) Cognitive neuroscience of emotional memory. Nat Rev Neurosci 7:54-64.

Lang PJ, Greenwald MK, Bradley MM, Hamm AO (1993) Looking at pictures: affective, facial, visceral, and behavioral reactions. Psychophysiology 30:261-273.

Liddell BJ, Brown KJ, Kemp AH, Barton MJ, Das P, Peduto A, Gordon E, Williams LM (2005) A direct brainstem-amygdala-cortical "alarm" system for subliminal signals of fear. Neuroimage 24:235-243.
Maratos EJ, Allan K, Rugg MD (2000) Recognition memory for emotionally negative and neutral words: an ERP study. Neuropsychologia 38: $1452-1465$.

McCullough A, Yonelinas AP (2011) The effects of post-encoding stress on recollection and familiarity for emotional and neutral images. Presented at the 18th Annual Meeting of the Cognitive Neuroscience Society, San Francisco, April.

McGaugh JL (2004) The amygdala modulates the consolidation of memories of emotionally arousing experiences. Annu Rev Neurosci 27:1-28.

Mondaca M, Hernández A, Pérez H, Valladares L, Sierralta W, Fernández V, Soto-Moyano R (2004) Alpha2-adrenoceptor modulation of long-term potentiation elicited in vivo in rat occipital cortex. Brain Res 1021: 292-296.

Mosher CP, Zimmerman PE, Gothard KM (2010) Response characteristics of basolateral and centromedial neurons in the primate amygdala. J Neurosci 30:16197-16207.

Murty VP, Ritchey M, Adcock RA, LaBar KS (2010) fMRI studies of successful emotional memory encoding: a quantitative meta-analysis. Neuropsychologia 48:3459-3469.

Peelen MV, Fei-Fei L, Kastner S (2009) Neural mechanisms of rapid natural scene categorization in human visual cortex. Nature 460:94-97.

Peters S, Cleare AJ, Papadopoulos A, Fu CH (2011) Cortisol responses to serial MRI scans in healthy adults and in depression. Psychoneuroendocrinology 36:737-741.

Phan KL, Taylor SF, Welsh RC, Ho SH, Britton JC, Liberzon I (2004) Neural correlates of individual ratings of emotional salience: a trial-related fMRI study. Neuroimage 21:768-780.

Redondo RL, Morris RG (2011) Making memories last: the synaptic tagging and capture hypothesis. Nat Rev Neurosci 12:17-30.

Roozendaal B, McEwen BS, Chattarji S (2009) Stress, memory and the amygdala. Nat Rev Neurosci 10:423-433.

Schmidt SR, Saari B (2007) The emotional memory effect: differential processing or item distinctiveness? Mem Cognit 35:1905-1916.

Schupp HT, Flaisch T, Stockburger J, Junghöfer M (2006) Emotion and attention: event-related brain potential studies. Prog Brain Res 156:31-51.

Sharot T, Phelps EA (2004) How arousal modulates memory: disentangling the effects of attention and retention. Cogn Affect Behav Neurosci 4:294-306.

Sharot T, Yonelinas AP (2008) Differential time-dependent effects of emotion on recollective experience and memory for contextual information. Cognition 106:538-547.

Sharot T, Verfaellie M, Yonelinas AP (2007) How emotion strengthens the recollective experience: a time-dependent hippocampal process. PLoS One 2:e1068.

Smith SM, Vela E (2001) Environmental context-dependent memory: a review and meta-analysis. Psychon Bull Rev 8:203-220.

Sommer T, Gläscher J, Moritz S, Büchel C (2008) Emotional enhancement effect of memory: removing the influence of cognitive factors. Learn Mem 15:569-573.

Stark CE, Squire LR (2001) When zero is not zero: the problem of ambiguous baseline conditions in fMRI. Proc Natl Acad Sci USA 98:12760-12766.

Strange BA, Hurlemann R, Dolan RJ (2003) An emotion-induced retrograde amnesia in humans is amygdala- and beta-adrenergic-dependent. Proc Natl Acad Sci USA 100:13626-13631.

Suzuki WA (1996) Neuroanatomy of the monkey entorhinal, perirhinal and parahippocampal cortices: organization of cortical inputs and interconnections with amygdala and striatum. Semin Neurosci 8:3-12.

Talmi D, Schimmack U, Paterson T, Moscovitch M (2007a) The role of attention and relatedness in emotionally enhanced memory. Emotion 7:89-102.

Talmi D, Luk BTC, McGarry LM, Moscovitch M (2007b) The contribution of relatedness and distinctiveness to emotionally-enhanced memory. J Mem Lang 56:555-574.

Talmi D, Anderson AK, Riggs L, Caplan JB, Moscovitch M (2008) Immediate memory consequences of the effect of emotion on attention to pictures. Learn Mem 15:172-182.

Tessner KD, Walker EF, Hochman K, Hamann S (2006) Cortisol responses of healthy volunteers undergoing magnetic resonance imaging. Hum Brain Mapp 27:889-895.

Törnqvist E, Månsson A, Larsson EM, Hallström I (2006) It's like being in 
another world-patients' lived experience of magnetic resonance imaging. J Clin Nurs 15:954-961.

Tully K, Bolshakov VY (2010) Emotional enhancement of memory: how norepinephrine enables synaptic plasticity. Mol Brain 3:15.

Tzourio-Mazoyer N, Landeau B, Papathanassiou D, Crivello F, Etard O, Delcroix N, Mazoyer B, Joliot M (2002) Automated anatomical labeling of activations in SPM using a macroscopic anatomical parcellation of the MNI MRI single-subject brain. Neuroimage 15:273-289.

Van Bockstaele EJ, Bajic D, Proudfit H, Valentino RJ (2001) Topographic architecture of stress-related pathways targeting the noradrenergic locus coeruleus. Physiol Behav 73:273-283.

van Stegeren AH, Goekoop R, Everaerd W, Scheltens P, Barkhof F, Kuijer JP, Rombouts SA (2005) Noradrenaline mediates amygdala activation in men and women during encoding of emotional material. Neuroimage 24:898-909. van Stegeren AH, Roozendaal B, Kindt M, Wolf OT, Joëls M (2010) Interacting noradrenergic and corticosteroid systems shift human brain activation patterns during encoding. Neurobiol Learn Mem 93:56-65.

Vuilleumier P (2005) How brains beware: neural mechanisms of emotional attention. Trends Cogn Sci 9:585-594.

Yonelinas AP (2001) Consciousness, control, and confidence: the 3 Cs of recognition memory. J Exp Psychol Gen 130:361-379.

Yonelinas AP, Kroll NE, Dobbins I, Lazzara M, Knight RT (1998) Recollection and familiarity deficits in amnesia: convergence of remember-know, process dissociation, and receiver operating characteristic data. Neuropsychology 12:323-339.

Yonelinas AP, Parks CM, Koen JD, Jorgenson J, Mendoza SP (2011) The effects of post-encoding stress on recognition memory: examining the impact of skydiving in young men and women. Stress 14:136-144. 